\title{
On Perimeter Coverage in Wireless Sensor Networks with Minimum Cost
}

\section{K.-S. Hung}

Department of Electrical and Electronic Engineering,

The University of Hong Kong,

Pokfulam Road, Hong Kong

E-mail: kshung@eee.hku.hk

\section{K.-S. Lui*}

Department of Electrical and Electronic Engineering,

The University of Hong Kong,

Pokfulam Road, Hong Kong

E-mail: kslui@eee.hku.hk

* Corresponding author

\begin{abstract}
One of the major applications of sensor networks is tracking and surveillance. Very often, a single sensor is sufficient to monitor a single target. However, when the object is very large, several sensors have to work together to monitor the object continuously. In this paper, we study how to identify a set of sensors that can cover the perimeter of a large target with the minimum cost. We develop a novel distributed algorithm that requires fewer messages than existing mechanisms. Our algorithm can be extended to solve the problem when the sensor range is adjustable. We provide a formal proof of correctness and convergence time analysis of our algorithm. We further demonstrate the performance through extensive simulations.
\end{abstract}

Keywords: perimeter coverage; sensor networks; minimum cost; circular-arc graph; distributed algorithm.

Reference to this paper should be made as follows: Hung, K.-S. and Lui, K.S. (2010) 'On Perimeter Coverage in Wireless Sensor Networks with Minimum Cost', International Journal of Sensor Networks, Vol. , Nos., pp.

Biographical notes: Ka-Shun Hung did his BEng. (first class honors) in Electrical and Electronic Engineering from the University of Hong Kong, Hong Kong. He continued his MSc in Electrical Engineering from the Columbia University, New York, and then received his $\mathrm{PhD}$ in the Electrical and Electronic Engineering from the University of Hong Kong, Hong Kong. His research interests include perimeter coverage, trust, and security issues in wireless sensor networks.

King-Shan Lui received the B.Eng. and M.Phil. degrees in Computer Science from the Hong Kong University of Science and Technology. After receiving her PhD degree from the University of Illinois at Urbana-Champaign, USA, she joined the Department of Electrical and Electronic Engineering of the University of Hong Kong. Her research interests include network protocol design and analysis, sensor networks, and Quality-ofService issues.

\section{Introduction}

Wireless sensor networks have caught lots of attentions in recent years, because applications that are too dangerous for humans to operate could be performed by WSNs (Estrin et al. (2002)). In monitoring applications, small battery-powered sensor nodes are deployed in a large scale. Each sensor node can sense the environment up to a certain sensing range. Thus, each sensor can only cover a limited physical area and sensors usually cooperate to achieve a certain monitoring objective. The monitoring objective is usually transformed to a coverage problem. Area coverage and target coverage are are two common monitoring objectives widely studied (Cardei and $\mathrm{Wu}(2004))$. An area is under monitoring in area coverage that any event occurs inside the area should be discovered. In target coverage, a number of target objects are needed to be observed. 
In this paper, we study another coverage problem, called perimeter coverage, in which the perimeter of a large object has to be monitored. One typical application scenario is to monitor the entire perimeter of the white house so as to ensure its security. Each sensor is associated with a cost. To reduce the total cost for monitoring, we would like to identify a set of sensors that can cover the whole perimeter with the minimum cost. This problem is similar to the minimum weight circle-cover problem in a circular-arc graph. There are some centralized algorithms in the literatures(Andrews and Lee (1995); Atallah et al. (1995); Bertossi (1988); Ibarra et al. (1992)). Nevertheless, these algorithms do not tailor for the broadcast and distributed nature of wireless sensor networks. Previously, a distributed algorithm has been developed (Chow et al. (2007a)) to solve this problem. This mechanism suffers from a high message overhead because all the nodes passing through a reference point has to initiate the search. In this paper, we further enhance the distributed protocol by reducing the message overhead to be proportional to the size of network. We also provide a formal proof of correctness of our proposed algorithm.

Many existing networks assume that the sensing range of a sensor is fixed. However, recent research shows that adjustable sensing range is possible. It is generally assumed that the cost associated with a smaller range is smaller (Cardei and Du (2005); Cardei et al. (2005); Cardei et al. (2006); Dhawan et al. (2006); OPS (2009); Wang and Medidi (2007); Wu and Yang (2004); Zhou et al. (2004)). In view of this, we enhance our algorithm to consider also sensors with adjustable sensing range without increasing the message overhead.

The paper is organized as follows. In Section 2, we briefly discuss the related work in the literatures. Then, the network model and our distributed algorithms under the fixed sensing range scenario will be discussed in detail in Section 3 together with a formal proof of correctness. In Section 4, we present the modifications required to our distributed algorithm under the adjustable sensing range scenario. We carried out extensive simulations and the results are presented in Section 5. Finally, we conclude our schemes in Section 6 .

\section{Related Work}

In this section, we briefly discuss the related work on the coverage problems in wireless sensor networks, and the related work on the centralized algorithms solving the circle-cover problems in circular-arc graphs.

The two traditional coverage problems are area coverage and target coverage. Area coverage problem refers to the cover of the whole target area by the sensors. There are a number of variations, including single area coverage (Carle and Simplot-Ryl (2004); Cao et al. (2004); Gupta et al. (2003); Gupta et al. (2006)), multiple area coverage (e.g., k-coverage (Huang and Tseng (2003); Huang and Tseng (2005))) and fractional area coverage (Hung et al. (2007); Ye et al. (2006)), etc. On the other hand, target coverage problem refers to the cover of a certain target object or a number of target objects within a certain area. Various algorithms have been proposed to tackle the node placement (Kar and Banerjee (2003)) and energy issues (Cardei and Du (2005); Cardei et al. (2006); Thai et al. (2007)) on the target coverage problem. While most of the existing work assume that a target object is a point in the area, (Olteanu et al. (2008)) consider the situation in which a target may take up a certain space in the target area, and the target is detected once any portion of it falls within the sensing area of the sensor.

In this paper, we are specifically interested in the perimeter coverage problem. Unlike that of Olteanu et al. (2008) which aims at detecting a shaped target object, we study how to find a set of sensors to cover the whole perimeter of a shaped target object based on some selection criteria. One of the fundamental criteria is to find the set with the minimum amount of sensors distributively (Chow et al. (2007b); Hung and Lui (2010a)). This problem is very similar to finding a minimum circle-cover of a circular-arc graph, which studies how to cover the whole circle using as few arcs as possible. Circular-arc graph problems have been studied for quite a long time. The centralized algorithms for finding the minimum circle-cover are described in (Atallah and Chen (1989); Bertossi (1988); Lee and Lee (1984); Yu et al. (1989)).

Unlike these previous works, our main focus in this paper is to find the minimum weight circle-cover of a weighted circular-arc graph in a distributed manner. In (Bertossi (1988)), a parallel algorithm was proposed to tackle minimum weight circle-cover problem. This was known to be the first parallel algorithm proposed. Other than that, some parallel algorithms have been developed (Atallah et al. (1995); Ibarra et al. (1992)) but they are centralized in nature and do not exploit the wireless nature of sensor networks. Previously, a distributed algorithm was developed to find the set of nodes with minimum cost that covers the entire perimeter of the target object in a sensor network (Chow et al. (2007a)). This is also known to be the first distributed algorithm developed. Unfortunately, the message overhead of this algorithm can be very high. In this paper, we propose another approach that can reduce the message overheads and then provide a formal proof of correctness of our developed approach.

On the other hand, adjustable sensing range technique arouses more and more attentions recently. Many studies show that the network lifetime can be prolonged if the sensing range is reduced to lower the sensing cost (Cardei and Du (2005); Cardei et al. (2005); Cardei et al. (2006); Dhawan et al. (2006); Wu and Yang (2004)). It is widely assumed that energy consumed by a sensor is related to the sensing range of the sensor. The longer the range or the larger the sensing area, the more the energy is needed. The Osiris photoelectric sensors OPS (2009) available in the market support this 
assumption. Therefore, in our paper, we also consider the scenario where a sensor can adjust its sensing range and the cost of sensing is related to the sensing range.

\section{Distributed Minimum Cost Cover Algorithms under fixed sensing range scenario}

\subsection{Notations and Definitions}

Before we describe our distributed protocol, we define our problem in this section. Some of the notations are borrowed from Hung and Lui (2010a).

\subsubsection{Cover Range, Cover and Cost of a Cover}

The perimeter of the big object to be monitored can be in any irregular shape as long as it forms a loop. Each sensor can only monitor a continuous portion of the perimeter. The portion that a sensor can cover is the cover range of the sensor. How a sensor determines the range is application dependent and it is outside the scope of this paper. Interested readers are referred to (Lee and Aghajan (2006); McCormick et al. (2006); Tezcan and Wang (2008)).

As the perimeter forms a close loop, we need a reference point on the perimeter to represent the starting point and also the end point of the perimeter so that a cover range can be represented as $[x, y]$ according to the coordinate system used on the perimeter. A possible way to represent $x$ and $y$ is to use the distance on the perimeter from the reference point. In this paper, we follow the practice in the circular-arc graph problem that we use $\left[0^{\circ}, 360^{\circ}\right)$ to model the whole perimeter. The cover range of a sensor $i$ is represented as $\left[s_{i}, t_{i}\right]$ and $\left[s_{i}, t_{i}\right]$ spans in the clockwise manner as illustrated in Figure 1. It is worth noting that our protocols can be applied to any perimeter coordinate system that represents the portion a sensor can cover as a range. If sensor $i$ does not cover $0^{\circ}, s_{i}<t_{i}$; otherwise, $t_{i}<s_{i}$.

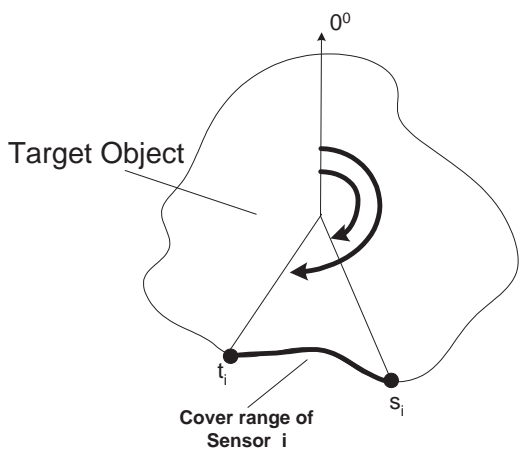

Figure 1 A sensor node $i$ with cover range $\left[s_{i}, t_{i}\right]$ on the perimeter of the target object.

Given a set of sensors $S$, a subset of sensors $D \subseteq S$ is a cover if for each angle $\gamma \in\left[0^{\circ}, 360^{\circ}\right)$, there exists a sensor $j \in D$ such that $\gamma \in\left[s_{j}, t_{j}\right]$. In other words, $\bigcup_{i \in D}\left[s_{i}, t_{i}\right]=\left[0^{\circ}, 360^{\circ}\right)$. Figure 2 illustrates a scenario of 9 sensors surrounding a target object, where each arrow represents the cover range of a node.

Each sensor $i$ is associated with a cost $f(i)$. In Figure 2, the number in circle represents the cost of each node. The cost of a cover $D, f(D)$, is the total cost of the sensors in the cover, that is, $f(D)=\sum_{i \in D} f(i)$. In the figure, the sets $\{1,3,5,7,8\},\{1,2,3,5,6,9\}$, and $\{1,3,5,7,9\}$ are all valid covers. The costs of the covers are 16, 22 and 17, respectively. Note that the cost of a sensor depends on the sensor application considered, and we do not specify any cost metric in this paper. Typical cost examples include the number of hop counts from the sensor to a sink for optimizing the message flow (Chow et al. (2007a)), or the remaining battery capacity of a sensor for extending the network lifetime (Chow et al. (2009)), or the cover range of a sensor for minimizing the amount of energy used for sensing (Hung (2010b)), etc.

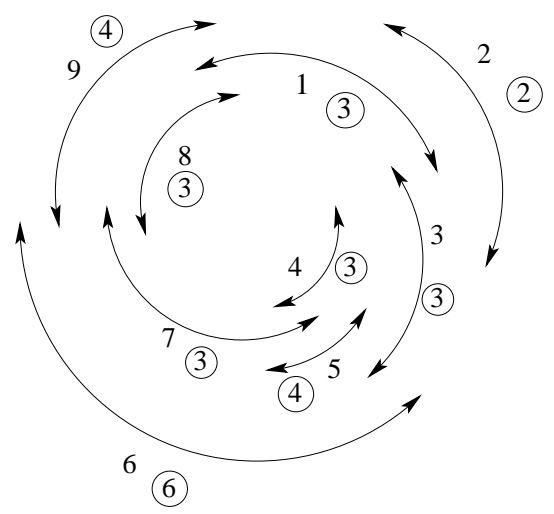

Figure 2 An example to illustrate the concept of minimum cost cover.

\subsubsection{Minimum Cost Cover}

Minimum Cost Cover (MCC) is a cover with minimum cost among all covers. Formally, $M$ is a minimum cost cover if $f(M) \leq f(D)$ for every cover $D \subseteq S$. For example, in Figure 2, $\{1,2,4,7,8\}$ is a Minimum Cost Cover, and the cost of the cover is 14 . We denote $M C C(i)$ to be a cover that contains $i$ while it is the minimum cost one among all covers that contain $i$. In other words, $f(M C C(i)) \leq f(D)$ for every cover $D$ where $i \in D$.

\subsubsection{Backward and Forward Neighbors}

Two nodes are neighbors if their cover ranges overlap. Formally, when $i$ and $j$ both do not cover $0^{\circ}, i$ and $j$ are neighbors if $s_{i}<s_{j}<t_{i}$ or $s_{i}<t_{j}<t_{i}$. The definition can be extended easily to ranges that cover $0^{\circ}$ but we leave it out for the ease of discussion. With the assumption that the communication range is twice of the sensing range, each node can communicate directly with neighbors only. It is possible that the sensing range of a sensor is completely contained in another one, like 
sensors 9 and 8 in Figure 2. When the sensing ranges of two sensors overlap and none of them is contained in the other, one sensor is a backward neighbor and the other sensor is a forward neighbor. $i$ is a backward neighbor of $j$ and $j$ is a forward neighbor of $i$ if $s_{i}<s_{j}<t_{i}$. Refer to Figure 2, sensors 2 and 3 are forward neighbors of sensor 1, while sensors 9 and 8 are backward neighbors of sensor 1 . As sensor 8 is completely contained in sensor 9 , they are not backward and forward neighbors of each other. It is worth noting that 8 and 9 would not appear together in the same minimum cost cover.

\subsection{Problem Statement}

In this paper, we aim at developing a distributed algorithm to find the minimum cost cover (MCC) of the perimeter of a large object which forms a loop. (Note that if the perimeter is not a loop, the problem is reduced to finding the set of sensors with minimum cost to cover a range, which can be solved in reasonable overhead (Chow et al. (2007a)).) Each node $i \in S$ with cover range $\left[s_{i}, t_{i}\right]$ can only communicate with neighbor nodes who have overlapping cover ranges. By communicating with neighbors only, when the algorithm terminates, $i$ can determine whether it is included in the MCC.

Theoretically, if we can find the cost of $M C C(i)$ for every $i \in S_{0}$, where $S_{0}$ contains the nodes with cover range passes through $0^{\circ}$, we know the cost of the minimum cost cover. Therefore, we first describe how to find $M C C(i)$.

\subsection{Finding $M C C(i)$}

When $i$ wants to identify $M C C(i)$, it initiates the search by sending a search message to all its forward neighbors. This message carries a cost value that keeps the total cost to cover the range starting from $s_{i}$ to the node that receives the message. When the message goes through the set of nodes in the clockwise direction, it will eventually be sent back to $i$ and $i$ will know which backward neighbor is in $M C C(i)$ and the cost of $M C C(i)$. This algorithm allows each node to determine whether it is in $M C C(i)$ or not but no node, even $i$, knows all the nodes that are in $M C C(i)$.

We now describe the mechanism in more detail and provide an example. When node $i$ wants to find $M C C(i)$, it sends the search message $\left\langle f(i), s_{i}, i\right\rangle$ to all its forward neighbors. When a node $j$ receives a message $<c, s_{i}, i>$ from its backward neighbor, it keeps track of the smallest $c$ it receives so far. After $j$ has received a message from each of its backward neighbors that starts on or after $s_{i}$, it sends $<c_{\text {min }}+f(j), s_{i}, i>$ to all its forward neighbors, where $c_{\text {min }}$ is the minimum $c$ among all the messages it received. Note that $j$ needs to receive the messages from backward neighbors that start after $s_{i}$ only. It is because nodes that start before $s_{i}$ will receive the search message later. Subsequently, each node will send out a search message, and $i$ will receive one search message from each of its backward neighbors. $i$ can then determine which backward neighbor is in $M C C(i)$.

We use an example to illustrate the mechanism. Suppose Node 1 in Figure 3 wants to find $M C C(1)$. Note that Node 0 is ignored in this example as it is contained in Node 1. It initiates the search by sending $<f(1)=3, s_{1}, 1>$ to all of its forward neighbors, i.e., Nodes 2 and 3. Node 2 has only one backward neighbor that starts on or after $s_{1}$, i.e., Node 1 . Therefore, Node 2 records $f(1)$ and sends $<f(1)+f(2)=7, s_{1}, 1>$ to its forward neighbors, which are Nodes 3 and 4 . Now, Node 3 has received a message from both its backward neighbors, 1 and 2. Definitely, it finds the cost value carried by the message sent by 1 is smaller and so it sends $<f(1)+f(3)=6, s_{1}, 1>$ to its forward neighbors 4 and 5 . This time, 4 receives messages from all its backward neighbors. Let the smaller cost among the messages that a node received be $c$. 4 sends $<c+f(4)=6+2=$ $8, s_{1}, 1>$ to its forward neighbors 5 and 6 . Subsequently, 5 sends out $\left\langle c+f(5)=6+1=7, s_{1}, 1>\right.$ and 6 sends out $\left\langle c+f(6)=7+2=9, s_{1}, 1>\right.$. Eventually, Node 1 would receive all messages from its backward neighbors and it can determine the cost of $M C C(1)=7$.

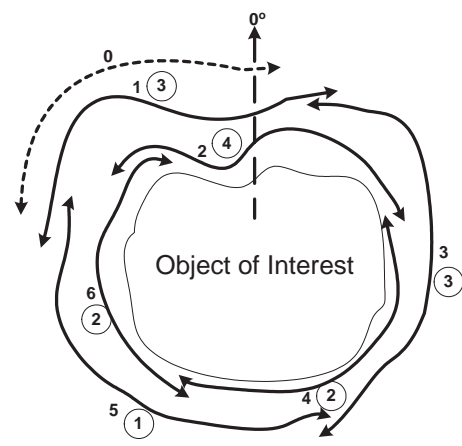

Figure 3 An $M C C(i)$ Search Algorithm Example.

The pseudocode of the $M C C(i)$ search algorithm of different states can be found in Algorithm 1 and Algorithm 2. When $i$ wants to start the search, it enters the INIT state. In this state, $i$ sends a search message to all its forward neighbors and then transits to the $W A I T$ state. For other nodes $n$, when they start up, they enter the INIT state. After initiating the variables in the INIT state, it transits to the WAIT state to wait for the search message from all its backward neighbors. Once it has received all backward neighbors' messages, it sends out the search message and enters FIN state. During this stage, node $n$ has completed its task in searching for $M C C(i)$ and so it waits for $<S E L E C T>$ message to see if it is selected in the $M C C(i)$. Once $i$ receives messages from all its backward neighbors, it can determine $M C C(i)$ by selecting the one with the minimum cost. Note that previous $(i)$ is used to store the id of the backward neighbor in which the search message with smaller cost comes from. At this stage, $i$ can enter $F I N$ state and send back the $<S E L E C T>$ message to 


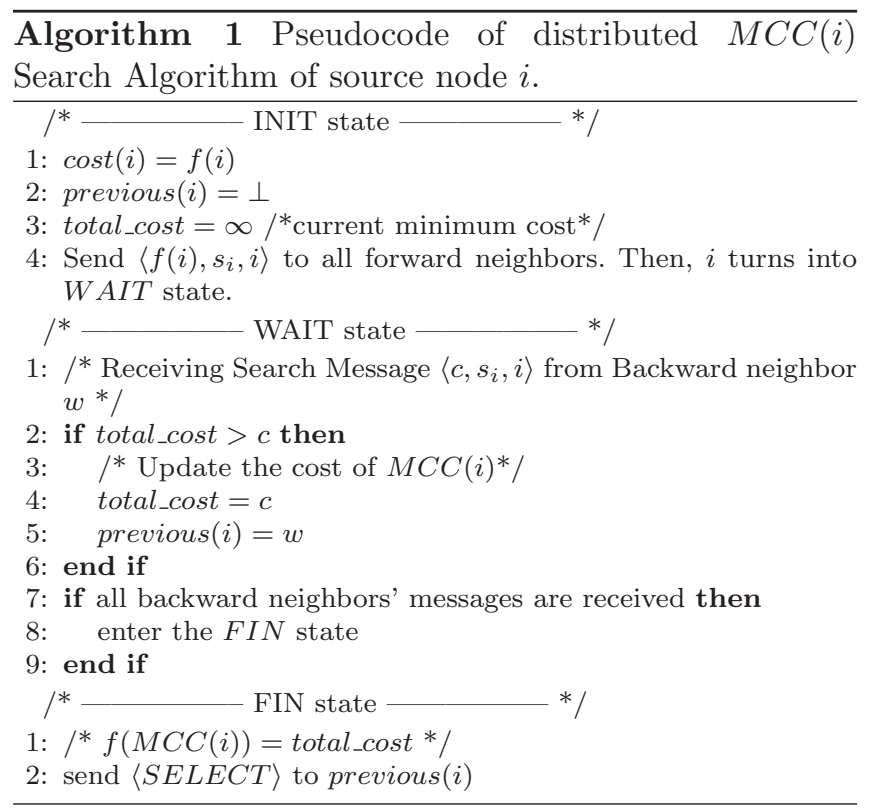

the node previous $(i)$ which denotes the node included in $M C C(i)$. When node $n$ overhears a $<S E L E C T>$ message and it is the recipient of the message, it realizes that it is selected in $M C C(i)$. Then, it further sends a $<S E L E C T>$ message to the node previous $(i)$. This process continues until the node previous $(i)$ is node $i$. Nodes that contain $i$ or contained in $i$ are not involved in the search. When they overhear the search message and realized that it is initiated by $i$, they do not have to participate the search.

Since each node needs to send a single search message and only those nodes selected to be in the cover need to send the $\langle S E L E C T>$ message, the message complexity of the algorithm is $O(N)$ where $N$ is the number of sensors in the network. We now formally prove the correctness of our mechanism.

\section{Lemma 3.1: $\quad M C C(i)$ Search Algorithm is correct.}

\section{Proof:}

To ease the discussion, we assume $s_{i}$ is $0^{\circ}$. We label the nodes according to their start angles. A node $a$ starts before $b$ if $s_{a}<s_{b}$. We also say $b$ starts after $a$. We label $i$, the node that initiates the search, as $n_{0}$ and the nodes start after $i$ as $n_{1}, n_{2}$, and so on where $n_{x}$ starts before $n_{y}$ if $x<y$. Note that apart from $n_{0}, n_{1}$ may have other backward neighbors. Nevertheless, $n_{1}$ does not have to receive messages from these backward neighbors before sending out its search message. We refer the backward neighbors whose search messages that a node needs to wait for as important backward neighbors. In general, the search messages that $n_{x}$ must receive before it sends out a search message must be from the nodes in the set $\left\{n_{0}, n_{1}, \ldots, n_{x-1}\right\}$. Besides, $n_{y}$ can never be an important backward neighbor of $n_{x}$ if $y>x$.

We prove this lemma by induction. $n_{0}$ initiates the search by sending $<f\left(n_{0}\right), s_{n_{0}}, n_{0}>$ to its forward neighbors. As $n_{0}$ is the only important backward neighbor of $n_{1}, n_{1}$ identifies the minimum cost $c$ and sends out the search message $<c, s_{n_{0}}, n_{0}>$ to its forward neighbors.

Assume that $n_{k}$ can identify the minimum cost from $n_{0}$ to itself. It should be noted that $n_{j}$ where $j<k$ should have sent out its message too. When $n_{k}$ sends out the search message, $n_{k+1}$ should have received a search message from each of its important backward neighbors since every node $n_{j}$ where $j \leq k$ has sent a message. Thus, $n_{k+1}$ can identify which important backward neighbor offers the minimum cost from $n_{0}$ to itself.

By induction, we prove that the minimum cost to reach $n_{k+1}$ can also be determined correctly after $n_{k}$ sends out its search message. Eventually, the search message will pass back to $n_{0}$, and $n_{0}$ can identify which backward neighbor should be included in the cover and no more search message is generated. Therefore, when the algorithm terminates, the cover $M C C(i)$ is identified. 


\subsection{Distributed Minimum Cost Cover Algorithm $(D M C C)$}

One way to find the minimum cost cover is to find the cost of each $M C C(i)$ where $i \in S_{0}$ and identify the minimum one. The overhead would be $O\left(\left|S_{0}\right| N\right)$ where there are $N$ sensors in the network. Since all nodes need to send out a search message for every individual $M C C(i)$ search, it is possible to reduce the overhead by "combining" the messages of several searches into a single message to reduce overhead. We now describe our DMCC algorithm that can identify the minimum cost cover with $O(N)$ number of messages in detail.

\subsubsection{Algorithm Description}

We call a node that covers $0^{\circ}$ a zero node. That is, every node in $S_{0}$ is a zero node. The main idea of our algorithm is that every node $u \in S$ does not send out a search message until it can identify the minimum cost from each zero node $i$ to itself. The search message sent by $u$ should contain information about all $M C C(i)$. The major challenges of this idea are: Who should initiate the algorithm? How should $u$ determine it has obtained all the necessary information to construct its search message? What should the search message look like? Can we prune the search of some $M C C(i)$ ? When will the algorithm terminate?

Combined Search Message Format: We first describe the search message. In the $M C C(i)$ search algorithm, each message is of format $\left\langle c, s_{i}, i\right\rangle$. The first element represents the cost, the second and the third values represent the start angle of the node that initiates the search and the identity of the node, respectively. To carry the minimum cost information of several searches in one message, we enhance the message to $<<c_{q_{1}}, s_{q_{1}}, q_{1}>, \ldots,<c_{q_{j}}, s_{q_{j}}, q_{j}>, \ldots,<$ $c_{q_{\left|S_{0}\right|}}, s_{\left|S_{0}\right|}, q_{\left|S_{0}\right|}>>$ where $c_{q_{j}}$ represents the minimum cost to reach the current node from the zero node $q_{j}$.

Message Combining Mechanism: We label the nodes in $S_{0}$ according to the ascending order of their start angles as $q_{1}, q_{2}, \ldots, q_{\left|S_{0}\right|}$. The nodes in $S_{0}$ are neighbors of each other since they all cover $0^{\circ}$. Therefore, by exchanging cover range messages, they can identify which node is $q_{1}$, which is $q_{2}$, etc.

The algorithm is best to be initiated by $q_{1}$. It is because if $q_{j}$, where $j \neq 1$, initiates the process, the process cannot combine the search message of $M C C\left(q_{k}\right), \forall k=1, \ldots, j-1$. In this case, the number of messages to find MCC will increase as extra messages are needed to carry the search of $M C C\left(q_{k}\right), \forall k=$ $1, \ldots, j-1$. The implication of this is that the number of messages required is minimum if the process is initiated by $q_{1}$. $q_{1}$ initiates the search by sending out $<f\left(q_{1}\right), s_{q_{1}}, q_{1}>$. Based on Lemma 3.1, we know that $q_{2}$ knows the minimum cost to reach itself from $q_{1}$ after receiving the search message of $q_{1}$. At this moment, it can combine $M C C\left(q_{1}\right)$ and $M C C\left(q_{2}\right)$. The combined message becomes $<<f\left(q_{1}\right)+f\left(q_{2}\right), s_{q_{1}}, q_{1}>,<f\left(q_{2}\right)$, $s_{q_{2}}, q_{2}>>$. The first tuple is used to continue the search of $M C C\left(q_{1}\right)$, while the second one is for searching $\operatorname{MCC}\left(q_{2}\right)$. Similar process will be carried out by $q_{3}, \ldots$, $q_{k}, \ldots, q_{\left|S_{0}\right|}$ accordingly. In other words, each node $q_{k} \neq$ $q_{1}$ has two tasks. First, it records the minimum costs from its backward neighbors in $S_{0}$ to itself. Second, it initiates the search of $M C C\left(q_{k}\right)$. After $q_{k}$ has received all the search messages from its important backward neighbors, it sends the combined search message to its forward neighbors. For all other non-zero nodes, they only have to carry out the first task.

Pruning Mechanism: It is obvious that if the combined message contains the information of the search of every zero node, we can identify the MCC finally. However, some of this information, in fact, can be pruned without affecting the correctness of the mechanism. After $n$ has received the search messages from all its backward neighbors, $n$ compares the costs among them. Let < $c_{q_{i}}, s_{q_{i}}, q_{i}>$ and $<c_{q_{j}}, s_{q_{j}}, q_{j}>$ be two tuples received by $n$. If $q_{i}$ starts earlier than $q_{j}\left(s_{q_{i}} \leq s_{q_{j}}\right)$ and $c_{q_{i}} \leq c_{q_{j}}$, $M C C\left(q_{j}\right)$ would not be the only minimum cost cover and $n$ can prune the search of $M C C\left(q_{j}\right)$. $n$ then sends the combined message which contains only the unpruned searches to all its forward neighbors.

Lemma 3.2: $\quad M C C$ will not be pruned by $n$.

\section{Proof:}

As $q_{i}$ starts earlier, the range covered by $q_{j}$ to $n$ $\left(\left[q_{j}, n\right]\right)$ must be contained in the range from $q_{i}$ to $n\left(\left[q_{i}, n\right]\right)$. Since $c_{q_{i}} \leq c_{q_{j}}$, if we replace the sensors in $M C C\left(q_{j}\right)$ that cover $\left[q_{j}, n\right]$ by the sensors in $\operatorname{MCC}\left(q_{i}\right)$ that cover $\left[q_{i}, n\right]$, this new cover must have a cost not larger than $M C C\left(q_{j}\right)$. Therefore, $n$ does not have to continue the search of $M C C\left(q_{j}\right)$.

Search Termination: Based on Lemma 3.1, we know that when $i$ initiates the search of $M C C(i)$, the search can be terminated once the message comes back to $i$ through a set of nodes in the clockwise direction. Recall that zero nodes $\left\{q_{1}, q_{2}, \ldots, q_{\left|S_{0}\right|}\right\}$ are sorted according to their start angles. Since our algorithm is initiated by $q_{1}$, through a set of nodes in the clockwise direction, the combined search message will eventually come back to $q_{1}$. At this moment, the cost of $M C C\left(q_{1}\right)$ can be determined. Instead of terminating the algorithm, $q_{1}$ has to continue all the remaining unpruned searches and inform other zero nodes the cost of $M C C\left(q_{1}\right)$. Again, two tasks are performed by a zero node $q_{k}$ once it has received all the search messages. First, $q_{k}$ terminates the search of $M C C\left(q_{k}\right)$, determines the cost of $M C C\left(q_{k}\right)$, and announces the cost to other zero nodes if $M C C\left(q_{k}\right)$ is unpruned. In fact, to determine whether $M C C\left(q_{k}\right)$ is pruned, $q_{k}$ can check if the search messages received still contains the entry about $M C C\left(q_{k}\right)$. Second, it continues the search of any unpruned $M C C\left(q_{l}\right)$, where $l>k$ by sending the combined message containing the tuple $<$ $c_{q_{l}}+f\left(q_{k}\right), s_{q_{l}}, q_{l}>$. Therefore, the whole search process can be terminated when $q_{\left|S_{0}\right|}$ can determine the cost 
of $M C C\left(q_{\left|S_{0}\right|}\right)$. Since all zero nodes can overhear each other, when $q_{\left|S_{0}\right|}$ announces the cost of $\operatorname{MCC}\left(q_{\left|S_{0}\right|}\right)$, all zero nodes should be able to determine which $M C C\left(q_{i}\right)$ is the smallest. $q_{i}$ can then inform its previous neighbor by a $<S E L E C T>$ message as described in Section 3.3.

Example: To better illustrate the algorithm, we describe an example. Refer back to Figure 3, Node 1 is $q_{1}$ and Node 2 is $q_{\left|S_{0}\right|}$. Node 1 initiates the DMCC algorithm by sending $\left\langle f(1), s_{1}, 1\right\rangle$ to its forward neighbors, in which $f(1)=3$ in our example. After Node 1 sends out the search message, Node 2 records the minimum cost to reach Node 2 from Node 1 as min_cost $(1)=f(1)+f(2)=3+4=7$. Since Node 2 is also a node in $S_{0}$, it will also initiate the search of $M C C(2)$. Therefore, Node 2 sends out $<<$ min_cost $(1)=7, s_{1}, 1>,<f(2)=4, s_{2}, 2>>$ to its forward neighbors. Then, when Node 3 receives the search message from Nodes 1 and 2, it records the minimum cost to reach Node 3 from Node 1 as min_cost $(1)=f(1)+f(3)=3+3=6$ and that of Node 2 as min_cost $(2)=f(2)+f(3)=4+3=7$. At this moment, Node 3 receives all the search messages from its backward neighbors. In this case, Node 3 can prune the search of $M C C(2)$ as Node 2 starts later than Node 1 and with the minimum cost larger than that of Node 1. Hence, Node 3 sends out $<$ min_cost $(1)=6, s_{1}, 1>$ to its forward neighbors. Then, Node 4 receives two search messages. They are $<4, s_{2}, 2>$ from Node 2 and $<6, s_{1}, 1>$ from Node 3 . It records the minimum cost to reach Node 4 from Node 1 and 2 as min_cost $(1)=$ $6+f(4)=6+2=8$ and min_cost $_{-}(2)=4+f(4)=4+$ $2=6$, respectively. Therefore, Node 4 sends out $<<$ min_cost $(1)=8, s_{1}, 1>,<\min _{-} \operatorname{cost}(2)=6, s_{2}, 2>>$ to its forward neighbors. Afterwards, Node 5 prunes the search of $M C C(2)$ and only sends out the search message of $M C C(1)$, i.e., $<\min \_\operatorname{cost}(1)=7, s_{1}, 1>$. Node 6 sends out the combine search messages for $M C C(1)$ and $M C C(2)$, i.e., $<<$ min_cost $(1)=7+2=9, s_{1}, 1>$ ,$<$ min_cost $(2)=6+2=8, s_{2}, 2>>$.

After receiving all the necessary search messages, Node 1 can determine the cost of $M C C(1)$ is 7 through Node 5. However, to complete the search of $M C C(2)$, Node 1 sends out $<$ min_cost $(2)=8+3=11, s_{2}, 2>$ to Node 2 together with the cost of $M C C(1)$. Node 2 then determines the cost of $M C C(2)=8$ through Node 6 and it informs Node 1 the cost. At this moment, the search process is terminated and Node 1 knows that $M C C(1)$ is the minimum cost cover.

In the previous discussion, we assume the zero node that starts the earliest initiates the algorithm. However, it is possible that a zero node does not have any zero node backward neighbors even it does not start the earliest. An example is Node 0 in Figure 3. In this case, this zero node should initiate the algorithm by itself.

Theorem 1: DMCC algorithm can find $M C C$ correctly.

\section{Proof:}

Since our mechanism combines the search messages without changing the method of finding $M C C(i)$ as discussed in Secton 3.3, Lemma 3.1 also implies the correctness of our mechanism except the pruning technique. Therefore, to prove the correctness of DMCC, we need to prove the correctness of the pruning technique. According to Lemma 3.2, we know that $M C C$ still exists in either one of the unpruned search of $M C C(i)$, where the search is on going. Hence, by exchanging the cost of all the unpruned $M C C(i)$, the minimum one is still MCC. Therefore, DMCC algorithm is correct.

DMCC algorithm requires every zero node to send out two search messages, while all other non-zero nodes need to send out one. This contributes to $O\left(N+\left|S_{0}\right|\right)$ number of messages. Since $\left|S_{0}\right| \leq N$, DMCC finds $M C C$ in $O(N)$ number of messages.

\section{Adjustable Sensing Range}

When describing DMCC above, we assumed sensors are using a single sensing range only. We now describe how DMCC can be enhanced to find the minimum cost cover when sensors can adjust their sensing ranges to several different levels in which a longer range will result in a higher cost.

\subsection{Problem Statement}

Each node can adjust its sensing range to different levels. Different nodes can have different numbers of levels and different sets of sensing ranges. Recall that $S$ denotes the set of sensors in the network. A sensor $i \in S$ can adjust its sensing range to $r$. This can be represented as an instance $i . r$ in the network. The set of all possible instances of sensors which are able to cover the perimeter of the target object is denoted as $S^{\prime}$. The cover range of an instance i.r $\in S^{\prime}$ is $\left[s_{i . r}, t_{i . r}\right]$ and the cost of this instance is $f(i . r)$. Under the adjustable sensing range environment, forward and backward neighbors concept defined in Section 3.1.3 applies to the instances of nodes instead of the nodes themselves. Given a cover $I$, if $i . r \in I, i . r^{\prime} \notin I$ for all possible sensing ranges $r^{\prime} \neq r$ of node $i$. The cost of a cover $I, f(I)$, is the total cost of the instances in the cover, that is, $f(I)=\sum_{i . r \in I} f(i . r)$. Suppose $D$ denotes the set of all possible covers. $M C C \subseteq$ $D$, such that $\forall I \subseteq D, f(M C C) \leq f(I)$.

In this scenario, our goal is to develop a distributed algorithm in which a node can identify whether any of its instance is included in the MCC.

\subsection{Adjustable Distributed Minimum Cost Cover algorithm (ADMCC)}

One of the major differences between the adjustable sensing range scenario and the fixed sensing range 
scenario is that in the fixed sensing range situation, every subset in $S$ is a valid selection. In contrast, some subsets in $S^{\prime}$ are not allowed since at most one instance of a node can be included in the cover. Fortunately, due to the properties among instances, our DMCC algorithm can be applied in $S^{\prime}$ with little modification.

\subsubsection{DMCC under adjustable sensing range}

Property 1: Suppose that $r$ and $r^{\prime}$ are two different sensing ranges of $i$ where $i \in S$. If $r<r^{\prime}$, then $i . r \in S^{\prime}$ is contained inside $i . r^{\prime} \in S^{\prime}$.

Property 1 shows that the instance of node $i$ with the smaller sensing range is contained inside the instance of the same node with the larger sensing range. This property is illustrated in Figure 4.

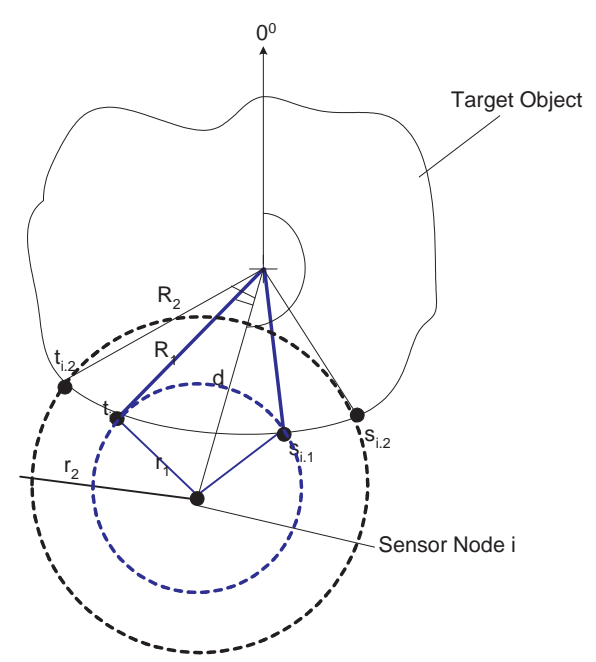

Figure 4 Adjustable Sensing Range Example

Since MCC would not contain two sensors where one is contained in the other, we have the following property.

Property 2: An MCC of $S^{\prime}$ does not contain both i.r and $i . r^{\prime}$ for any $i \in S$ and two different sensing ranges $r$ and $r^{\prime}$.

Due to Property 2, DMCC can still be directly applied to $S^{\prime}$ with a minor modification that a node $i \in S$ has to send and receive messages on behalf of its instances i.r $\in S^{\prime}$.

\subsubsection{Description of ADMCC}

Unfortunately, applying DMCC to $S^{\prime}$ directly will incur $O\left(\left|S^{\prime}\right|\right)$ messages. Therefore, the overhead is increased by a factor of the number of sensing levels. To reduce overhead, a node sends out a combined message for all its instances instead of sending one message for each instance.

Initiator Node: Recall that, in DMCC, the zero node that starts earliest or a zero node that does not have a zero backward neighbor should initiate the search.
However, the node with the zero instance (an instance that covers $0^{\circ}$ ) in $S^{\prime}$ that starts earliest may not be the appropriate node to initiate the search if we would like to combine the message for all its instances. Consider the configuration in Figure 5. j.r $r_{1}$ is the zero instance that starts the earliest. If $j$ initiates the computation process, it also sends out a search message for $M C C\left(j . r_{2}\right)$. It is not correct since $j . r_{2}$ is a forward neighbor of $i . r_{1}$ and violates the condition in DMCC that a node should not send out a search message until it has received the search messages from all its zero backward neighbor instances. Before we describe which node should start the search, we first define several definitions.

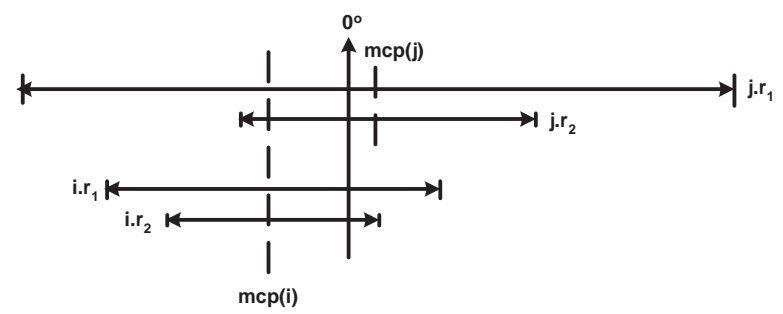

Figure 5 An example of $m c p(i)<m c p(j)$.

In the fixed range situation, we call a node zero node if it covers $0^{\circ}$. In the adjustable sensing range case, a node $i$ is a zero node if there exists an instance of $i$ that covers $0^{\circ}$. It is possible that not all instances of a zero node cover $0^{\circ}$. On the other hand, it is possible for a node $i$ to have all its instances share a common mid-point as shown in Figure 4. Note that this assumption may not be true if the target object is in irregular shape. In this case, we only combine those instances that share a common midpoint. The mid-cover-point of node $i$, denoted as $m c p(i)$, is the center of its cover ranges in different instances. For any $i, j \in S$, we define $m c p(i)<m c p(j)$ if $m c p(i)$ is on the left of $m c p(j)$ along the circle as shown in Figure 5. We have the following property:

Property 3: For any $i, j \in S$, if $\operatorname{mcp}(i)<m c p(j)$, then i.x would not be a forward neighbor of $j . y$ for any sensing level $x$ and $y$.

In the example shown in Figure 5 where $i$ and $j$ are both zero nodes, we say $m c p(i)<m c p(j)$ since $m c p(i)$ is to the left of $m c p(j)$. To show this property, suppose that there is an instance $i . x$ which is a forward neighbor of an instance $j . y$. That is, $s_{j . y}<s_{i . x}$. Since $m c p(j)$ is the mid-point of the cover range, $t_{j . y}=\operatorname{mcp}(j)+\operatorname{mcp}(j)-$ $s_{j . y}$. Similarly, $t_{i . x}=2 * m c p(i)-s_{i . x}$. Since $m c p(i)<$ $m c p(j)$ and $s_{j . y}<s_{i . x}$, we have $t_{i . x}<t_{j . y}$, which means $i . x$ is contained in $j . y$. It leads to contradiction since $j . y$ is not a forward neighbor of $i . x$ according to our definition in Section 3.1.3.

Property 3 suggests that we should select a zero node with the smallest mid-cover-point to initiate the algorithm since the zero instances of this node would not be forward neighbors of other zero instances. We thus select node $i$ to start the algorithm where node $i$ satisfies: 
1. $i$ is a zero node

2. $m c p(i)<m c p(j)$ for any zero node $j$ (Note that $x<y$ means $x$ is to the left of $y$ along the circle.)

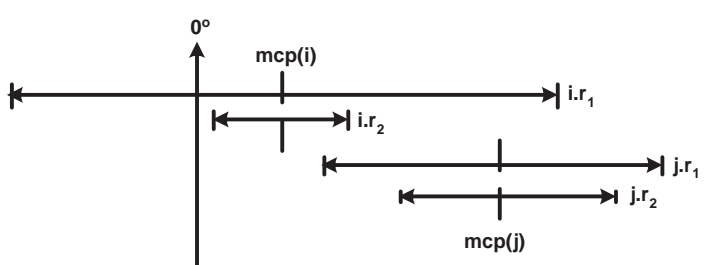

Figure 6 An initiator with both zero and non-zero instances.

We mentioned earlier that not all instances of a zero node are zero instances as well. Therefore, it is possible that the initiator node $i$ contains an instance that does not cover $0^{\circ}$ as shown in Figure 6 . In this case, $i$ should only carry the search information of those zero instances. In fact, if the non-zero instance of $i$ is to the right of $0^{\circ}$, it can be pruned ( $i r_{2}$ in Figure 6$)$. It is because a backward neighbor of this non-zero instance must cover $0^{\circ}$, which implies that there exist another zero node with mid-cover-point smaller than $m c p(i)$. However, this is not possible since $m c p(i)$ is the smallest. Hence, $i$ is selected to start the algorithm.

Message Combining Mechanism: After node $i$ sends out the search message that carries the information of $M C C(i . r)$ for every zero instance i.r, other nodes send out a combined search message after collecting a search message for each backward neighbor of each of its instances. We now explain there will be no deadlock in the whole procedure. That is, once the search has been started by $i$, every node will eventually receive enough messages from its backward neighbors and create its own search message.

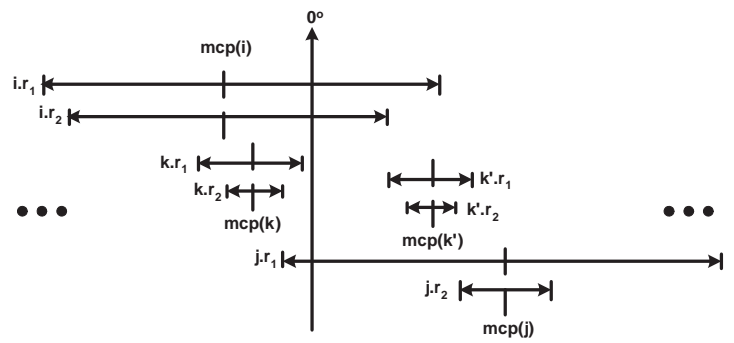

Figure 7 Order of nodes in ADMCC

Lemma 4.1: Every node will eventually send out a search message in the ADMCC algorithm.

Proof:

We label the nodes as $n_{0}, n_{1}, \ldots, n_{|S|}$. Let the initiator node be $n_{0}$. There are two cases:

1. $\operatorname{mcp}\left(n_{0}\right)$ is to the right of $0^{\circ}$

In this case, all the non-zero nodes $k$ with $0<$ $m c p(k)<m c p\left(n_{0}\right)$ can be pruned. We arrange the nodes according to the mid-cover-points. That is, $\operatorname{mcp}\left(n_{x}\right)<\operatorname{mcp}\left(n_{y}\right)$ if $x<y$.

2. $\operatorname{mcp}\left(n_{0}\right)$ is to the left of $0^{\circ}$

In this situation, nodes are divided into two sets. Set $A$ contains the non-zero nodes $k$ with $m c p\left(n_{0}\right)<\operatorname{mcp}(k)<0^{\circ}$ while $B=S \backslash A$. Note that $n_{0} \in B$. We first arrange the nodes in $B$ according to the mid-cover-point and then the nodes in $A$. For example, the nodes are arranged in Figure 7 according to the following order of mid-cover-points, i.e., $\operatorname{mcp}(i), \operatorname{mcp}\left(k^{\prime}\right), \operatorname{mcp}(j), \ldots$, $\operatorname{mcp}(k)$.

By defining the order in this way, Property 3 ensures that the important backward neighbors of the instances of node $n_{x}$ must be instances of nodes in the set $\left\{n_{0}, n_{1}, \ldots, n_{x-1}\right\}$.

We prove this lemma by induction. $n_{0}$ initiates the search by sending one combined search message for all its zero instances to its forward neighbors. If all the instances of $n_{0}$ are zero instances as shown in Figure $5, n_{1}$ has received a search message from each of the important backward neighbors of its instances. If NOT all $n_{0}$ 's instances cover $0^{\circ}$, the non-zero instances are either all to the left of $0^{\circ}$ or all to the right of $0^{\circ}$. If they are on the right, they have been pruned as in Figure 6 and should not be considered by $n_{1}$. If they are on the left, they are not important backward neighbors as defined in the proof of Lemma 3.1 and need not be considered as well. Therefore, we can conclude that $n_{1}$ has received all the search messages from all of its important backward neighbors and it can send out its search message.

Assume that $n_{k}$ has received all the search messages on behalf of all its instances. It should be noted that $n_{j}$ where $j<k$ should have sent out its combined search message too. When $n_{k}$ sends out the combined search message, $n_{k+1}$ should have received a combined search message from each of its important backward neighbors and it can send out its own search message. Therefore, there is no deadlock in the algorithm.

By Lemma 4.1, we know that a node $i$ can send out one combined search message for all its instances after it has received messages from all their backward neighbors. By combining the search messages of all its instances, the number of messages can effectively be reduced to $O(N)$.

Search Termination: In ADMCC, when a zero node $q_{i}$ receives all the search messages for all its instances, it can determine the cost of all unpruned $M C C(a)$, where a represents a zero instance of $q_{i}$. Instead of terminating the algorithm, it still has to continue the search of the remaining unpruned $M C C(b)$, where $b$ is another zero instance of the zero node $q_{k}$ and $\operatorname{mcp}\left(q_{i}\right)<\operatorname{mcp}\left(q_{k}\right)$. At the same time, $q_{i}$ announces the costs of all the unpruned $M C C(a)$. The whole search process can be terminated when the zero node with the largest mid-cover-point receives search messages for 
all its zero instances and then announces the costs. At this moment, the costs of all unpruned searches can be determined. By overhearing others' announcements, each zero node knows which instance is selected to form the minimum cost cover.

Search Message Format: In the combined message, we need to identify the searches of different instances of different nodes. In ADMCC, the search message format is $<<e_{1},<$ combined search message of instance $e_{1}>>,<e_{2},<$ combined search message of instance $e_{2}>>, \ldots>$ where $e_{k}$ is an instance of the node which sends out the combined search message. The format of the combined search message of instance $e_{k}$ is similar to the combined search message of the node in DMCC defined in Section 3.4.1.

Example: As shown in Figure 8, Node 1 has two zero Instances 1.1 and 1.2. Since Node 1 is the only zero node, it initiates the process of computing the minimum cost cover. Hence, Node 1 sends $<<1.1,<f(1.1)=$ $5, s_{1.1}, 1.1>>,<1.2,<f(1.2)=2, s_{1.2}, 1.2>>>\quad$ to all the forward neighbors. After receiving the search message from Node 1, Node 2 updates the minimum cost to reach Instance 2.1 from Instance 1.2 as $c_{-} \min _{1.2,2.1}=$ $f(1.2)+f(2.1)=5+2.5=7.5$. It also determines the minimum cost to reach Instance 2.2 from Instance 1.1 as $c_{-}$min $_{1.1,2.2}=f(1.1)+f(2.2)=2+6=8$ and that from Instance 1.2 as $c_{-}$min $_{1.2,2.2}=f(1.2)+f(2.2)=$ $5+6=11$. Since Node 2 has received the search messages of all of its instances (i.e., all the backward neighbors of its instances), Node 2 sends the combined message $\quad<<2.1,<c_{\_} \min _{1.2,2.1}=7.5, s_{1.2}, 1.2>,<$ $2.2<c_{-} \min _{1.1,2.2}=8, s_{1.1}, 1.1>,<c_{-} \min _{1.2,2.2}=$ $11, s_{1.2}, 1.2>>>$ to the forward neighbors.

Upon receiving the search message from Node 2, Node 3 determines the minimum cost to reach Instance 3.1 from zero Instances 1.1 and 1.2 as $c_{-}$min $_{1.1,3.1}$ $=c_{-} \min _{1.1,2.2}+f(3.1)=8+3=11$ and $c_{-}$min $_{1.2,3.1}$ $=\quad c_{-} \min _{1.2,2.2}+f(3.1)=11+3=14$, respectively. Moreover, it determines the minimum cost to reach Instance 3.2 from Instances 1.1 and 1.2 as $c_{-} \min _{1.1,3.2}$ $=c_{-} \min _{1.1,2.2}+f(3.2)=8+8=16$ and $c_{\_} \min _{1.2,3.2}=$ c_min $_{1.2,2.2}+f(3.2)=11+8=19$, respectively. At this moment, Node 3 has received all the search messages to make its own. It sends $<<3.1,<c_{-}$min $_{1.1,3.1}=11$, $s_{1.1}, 1.1>,<c_{-} \min _{1.2,3.1}=14, s_{1.2}, 1.2>>,<3.2,<$ $c_{-} \min _{1.1,3.2}=16, s_{1.1}, 1.1>,<c_{-} \min _{1.2,3.2}=19, s_{1.2}$, $1.2>>>$ to the forward neighbors of its instances. Once Node 1 receives the combined search message from Node 3. It determines the cost of $M C C(1.2)$ is $c_{-} \min _{1.2,3.1}=14$, while the cost of $M C C(1.1)$ is $c_{-} \min _{1.1,3.2}=16$. Since Node 1 is the only zero node, the cost of $M C C$ can be determined once the costs of $M C C(1.1)$ and $M C C(1.2)$ are determined. The one with the minimum cost is $\mathrm{MCC}$ which is $M C C(1.2)$.

We refer readers to (Hung and Lui (2008)) for a complete descriptions of pseudocodes and state diagrams.

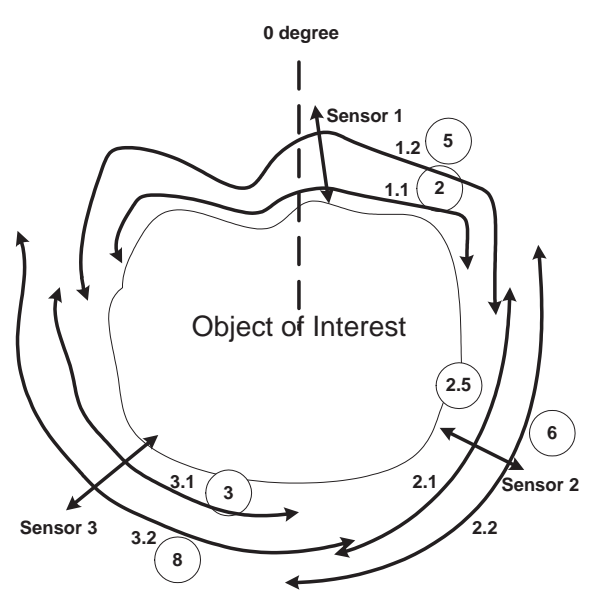

Figure 8 Adjustable Distributed Minimum Cost Cover Algorithm Example

\section{Simulation}

For comparison, we have implemented the algorithm described in (Chow et al. (2007a)) and our proposed algorithms (DMCC and ADMCC) in this paper. In the algorithm described in (Chow et al. (2007a)), each zero node $i$ finds $M C C(i)$ individually and there is no combining of messages. We denote this algorithm as Exhaustive Distributed Minimum Cost Cover Algorithm (Ex-DMCC) in this literature.

\subsection{Simulation Settings}

Our simulation environment is similar to the one adopted in (Chow et al. (2007b)). We considered an area of 100 grids $\times 100$ grids, in which each grid takes up an area of 1 unit $^{2}$ and every grid has a probability of 0.8 to contain a sensor. The average number of sensors is around 6500 . The target object, which is a circle with radius of 25 units, is located at the center $(50,50)$. A sensor can cover the portion of the perimeter of the target if that portion is within the sensing range of the sensor. We measure the performance of our mechanisms for different sensing range values. The cost value associated with each node is randomly generated from $1-2$. Under the adjustable sensing range scenario, we have $f(i . r) \propto r^{2}$ (Cardei and Du (2005); Cardei et al. (2005); Cardei et al. (2006)). We randomly generated 30 topologies for each set of results. Four performance metrics are measured and they are the total number of messages generated, the expected energy expenditure due to the transmission of messages, the minimum cost of the covers found by the algorithm, and the total time needed to find MCC.

\subsection{Simulation Results}

\subsubsection{Fixed Sensing Range Scenario}

We compare the message overheads and energy needed to find the minimum cost cover using Ex-DMCC and 
DMCC to show the effect of combining messages. Figure 9 illustrates the log number of messages to find the covers with different fixed sensing ranges. The figure shows that Ex-DMCC requires a large amount of messages to find the MCC. This is because it requires each zero node $q_{i}$ to look for $M C C\left(q_{i}\right)$ individually and $\left|S_{0}\right|$ can be large in some situations. On the other hand, DMCC greatly reduces the message overhead by combining the messages, so that most of the nodes only need to send out the combined search message once. Note the message overheads of DMCC with or without pruning are similar. Therefore, we show only the results with both combined messages and pruning in Figure 9. On the other hand, both algorithms show an increase in the number of messages with an increase in the sensing range. It is because when the sensing range is short, some sensors may not be able to cover the target perimeter and these sensors are not involved in the protocol. It can be observed that the overhead in Ex-DMCC increases faster than that of DMCC when the sensing range increases. This is because when nodes have a larger sensing range, more nodes would cover $0^{\circ}$ and so $\left|S_{0}\right|$ is larger.

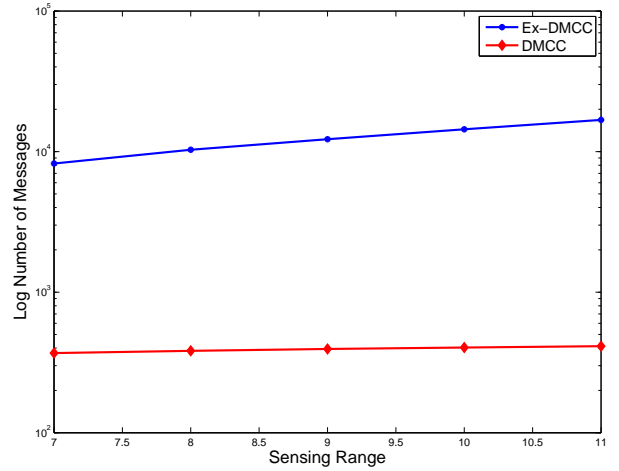

Figure 9 Log Number of Messages Vs. Sensing Range.

Other than message overheads, energy needed to find the minimum cost cover is also our concern. In fact, each Ex-DMCC message contains three tuples $\left\langle c, s_{i}, i\right\rangle$, while each DMCC message contains several combined Ex-DMCC messages. Therefore, the size of a DMCC message can be multiple times of that of an Ex-DMCC message. Practically, $c$ and $s_{i}$ can be rounded to 1 byte integers with a certain loss of precision. $i$ depends on the size of the network and it can be represented by an integer smaller than 2 bytes in general. Therefore, the size of an Ex-DMCC message is around 4 bytes, while that of DMCC message is around $\left|S_{0}\right| \times 4$ bytes if none of the search is pruned. In (Shnayder et al. (2004); Shnayder et al. (2009)), the authors suggested that CSMA should be performed before sending a message so as to avoid collision. The average CSMA time is around $41.0 \mathrm{~ms}$. On the other hand, we know that transmitting with the maximum range incurs a current of $21.5 \mathrm{~mA}$, while listening to the channel incurs a current of $7 \mathrm{~mA}$. In other words, the transmitting and the listening operations incur a power of $64.5 \mathrm{~mW}$ and
$21 \mathrm{~mW}$ under a $3 \mathrm{~V}$ power supply. The transmission rate is around 16kbps. The energy consumed for sending a message can be estimated by $P_{t} \times T_{x}+P_{i} \times I_{x}$, where $P_{t}$ and $P_{i}$ are the power required for transmitting and listening, respectively. $T_{x}$ is the time durations required for transmitting the whole message and can be estimated by $\frac{\text { message size }}{\text { transmission rate }}$, while $I_{x}$ is the average CSMA time. From the simulation, we know that $\left|S_{0}\right|$ is generally less than 50 after pruning. Therefore, a combined message contains approximately 200 bytes only. Based on the information above, the energy consumed for transmitting a 4-byte message and a 200-byte message are $1 \mathrm{~mJ}$ and $7.3 \mathrm{~mJ}$, respectively. Figure 10 illustrates the estimated transmission energy expenditure of ExDMCC, DMCC with the combining technique but without the pruning technique, and DMCC with both the combining technique and the pruning technique. The figure shows that DMCC with both the combining and the pruning techniques uses up the least amount of energy. This is mainly due to the reason that DMCC requires much fewer messages and smaller message size to find the minimum cost cover.

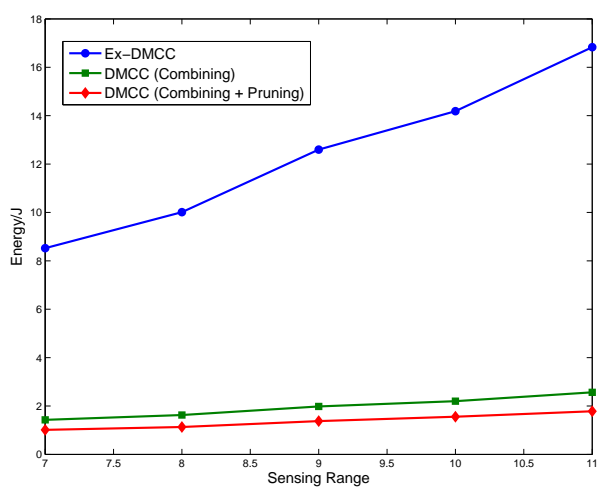

Figure 10 Estimated Transmission Energy Expenditure Vs. Sensing Range.

The total time needed to find MCC can be estimated by finding the total CSMA time and the total transmission time. These two parameters can be found by using the transmission rate, the mean CSMA time information, and the average size of a message. Note that we assume all $q_{i} \in S_{0}$ initiate the search of $\operatorname{MCC}\left(q_{i}\right)$ simultaneously in the Ex-DMCC algorithm. Figure 11 illustrates the estimated total time needed of Ex-DMCC, DMCC with the combining technique but without the pruning technique, and DMCC with both the combining technique and the pruning technique. The figure shows that DMCC with both the combining and the pruning techniques is the fastest in finding MCC. This is mainly due to the reason that DMCC requires fewer messages (which affects the CSMA time) and smaller message size to find the minimum cost cover (which affects the transmission time). 


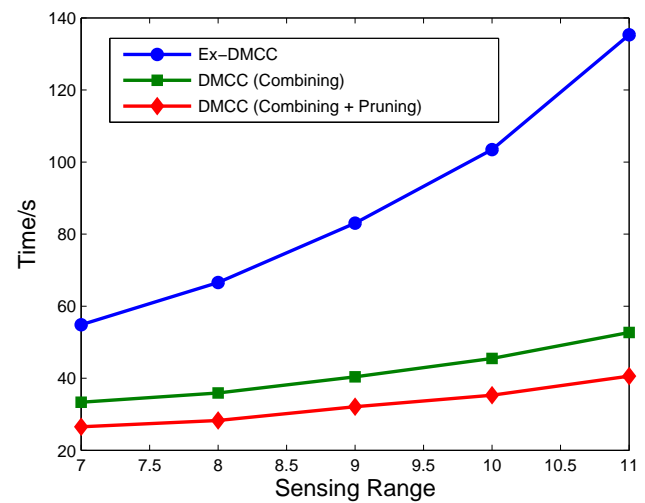

Figure 11 Estimated Total Time needed to find MCC Vs. Sensing Range.

\subsubsection{Adjustable Sensing Range Scenario}

In our simulations, we measure the performance of four situations which have different numbers of sensing levels. Particularly, 1SR, 2SR, 4SR, and 6SR represent one level, two levels, four levels, and six levels, respectively. We assume that there exists a maximum sensing range $r_{m}$. For $x \mathrm{SR}$, the sensing range $r_{i}=\frac{i \times r_{m}}{x}$ is associated with the cost $f(i)=f\left(r_{m}\right) \times\left(\frac{r_{i}}{r_{m}}\right)^{2}$, for $i=1$ to $x$. This arrangement is based on the settings in (Cardei et al. (2006); Chow et al. (2007a)).

Figure 12 shows that the message overhead produced by DMCC increases as the number of the sensing levels increases. In contrast, the message overhead of ADMCC, in which a node sends out a combined message for all its instances, changes only a little. Figure 13 shows

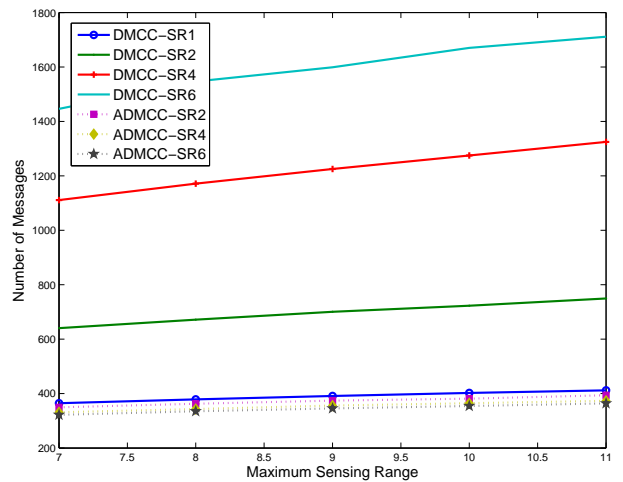

Figure 12 Number of Messages Vs. Maximum Sensing Range.

that the estimated transmission energy expenditure of both DMCC and ADMCC increases as the number of the sensing levels increases. ADMCC requires fewer messages than that of DMCC and this leads to smaller amount of transmission energy expenditure. Due to this reason, the energy expenditure differences between ADMCC and DMCC increases with the numbers of sensing levels as a lot of messages are required to find the minimum cost cover in DMCC, but nearly the same amount of messages are required to find the minimum cost cover in ADMCC.

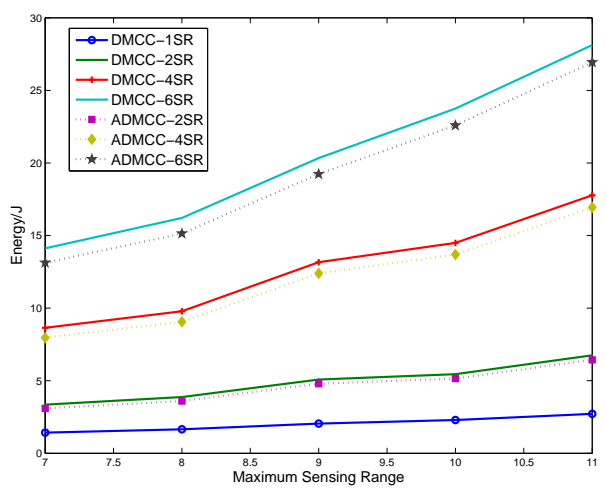

Figure 13 Estimated Transmission Energy Expenditure Vs. Sensing Range.

Figure 14 shows that the estimated total time needed of both DMCC and ADMCC increases as the number of the sensing levels increases. ADMCC requires fewer messages than that of DMCC and this leads to shorter total CSMA time. Due to this reason, the total time difference between ADMCC and DMCC increases with the numbers of sensing levels as a lot of messages are required to find the minimum cost cover in DMCC, but nearly the same amount of messages are required to find the minimum cost cover in ADMCC.

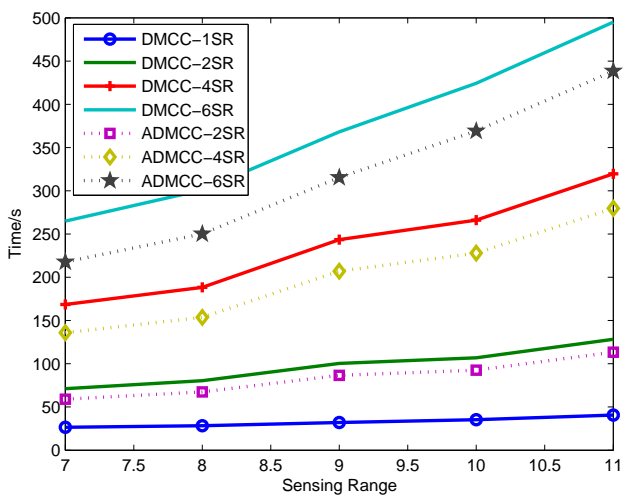

Figure 14 Estimated Total Time needed to find MCC Vs. Sensing Range.

Figure 15 illustrates the costs of the minimum cost cover of various maximum sensing range settings. As expected, the more the sensing levels, the smaller the cost of covers due to the reason that finer adjustment on sensing range can be applied to each node so as to cover the target object. However, the advantage in the cover costs becomes smaller when the number of sensing levels further increases. This is because the advantage brought by the finer adjustment of the sensing range becomes smaller and smaller. 


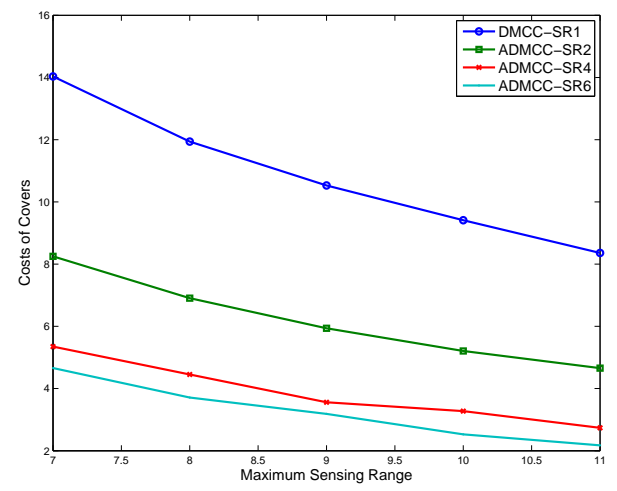

Figure 15 Cover Costs Vs. Maximum Sensing Range.

\section{Conclusion}

In this paper, we focus on the angle/perimeter coverage problem in which multiple sensors are expected to collaborate to monitor the whole perimeter of a large target object with the minimum cost. The algorithm is distributed in nature that when it terminates, every sensor can finally determine whether it is in the cover by communicating only with its neighbors. This coverage problem is very different from some existing coverage problems in which a certain area or point are to be monitored instead. To the best of our knowledge, we are the first to propose a distributed algorithm for finding the minimum cost cover with the communication complexity of $\mathrm{O}$ (size of the network). We also give the formal proof of correctness of our algorithm. Moreover, we are the first to study this problem under the adjustable sensing range scenario. Through extensive simulations, our proposed DMCC algorithm outperforms Ex-DMCC in terms of the communication overhead, and ADMCC can find a cover with smaller cost than the existing algorithms in the adjustable sensing range scenario.

\section{References}

Andrews, M. G. and Lee, D. T. (1995) 'Parallel algorithms on circular-arc graphs', Computational Geometry - Theory and Applications, Elsevier Science Publishers, 1995.

Atallah, M. J. and Chen, D. Z. (1989) 'An Optimal Algorithm for the Minimum Circle-Cover Problem', Information Processing Letters, Elsevier Science Publishers, vol. 32, 1989, pp. 159-165.

Atallah, M. J., Chen, D. Z. and Lee, D. T. (1995) 'An Optimal Algortihm for Shortest Paths on Weighted Interval and Circular-Arc Graphs, with Applications', Algorithmica, vol. 14, November, 1995, pp. 429-441.

Bertossi, A. A. (1988) 'Parallel Circle-Cover Algorithms', Information Processing Letters, Elsevier Science Publishers, vol. 27, 1988, pp. 133-139.

Cardei, M. and Wu, J. (2004) 'Coverage in Wireless Sensor Networks', Handbook of Sensor Networks, M. Ilyas and I. Magboub (eds.), CRC Press, 2004.
Cardei, M. and Du, D.-Z. (2005) 'Improving wireless sensor network lifetime through power aware organization', Wireless Networks, Kluwer Academic Publishers, vol. 11, No. 3, May, 2005, pp. 333-340.

Cardei, M., Wu, J., Lu, M., and Pervaiz, M.O.(2005) 'Maximum Network Lifetime with Adjustable Range', IEEE International Conference on Wireless and Mobile Computing, Networking and Communications (WiMob), August, 2005.

Cardei, M., Wu, J., Lu, M. (2006) 'Improving network lifetime using sensors with adjustable sensing range', International Journal of Sensor Networks, 2006, pp. 4149.

Carle, J. and Simplot-Ryl, D. (2004) 'Energy-efficient area monitoring for sensor networks', Computer, IEEE, Vol. 37, February, 2004, pp. 40-46.

Cao, G., Wang, G., La Porta, T., Phoha, S., Wang, G. and Zhang, W. (2004) 'Distributed algorithms for deploying mobile sensors', Theoretical and Algorithmic Aspects of Sensor, Ad Hoc Wireless and Peer-to-Peer Networks, 2004, pp. 427-439.

Chow, K.-Y., Lui, K.-S. and Lam, Edmund Y. (2007a) 'Achieving 360 Angle Coverage with Minimum Transmission Cost in Visual Sensor Networks', IEEE Wireless Communications and Networking Conference (WCNC), March, 2007, pp. 4112-4116.

Chow, K.-Y., Lui, K.-S. and Lam, Edmund Y. (2007b) 'Maximizing Angle Coverage in Visual Sensor Networks', IEEE International Conference on Communications (ICC 2007), June, 2007, pp. 3516-3521.

Chow, K.-Y., Lui, K.-S. and Lam, Edmund Y. (2009) 'Wireless sensor networks scheduling for full angle coverage', Multidimensional Systems and Signal Processing, Vol. 20, No. 2, June, 2009, pp. 101-119.

Dhawan, A., Vu, C.T., Zelikovsky, A., Li, Y. and Prasad, S. K. (2006) 'Maximum Lifetime of Sensor Networks with Adjustable Sensing Range', Proceedings of the Seventh ACIS International Conference on Software Engineering, Artificial Intelligence, Networking, and Parallel Distributed Computing, 2006.

Estrin, D., Culler, D., Pister, K. and Sukhatme, G. (2002) 'Connecting the physical world with pervasive networks', Pervasive Computing, IEEE, vol. 1, Jan-Mar, 2002, pp. 59-69.

Gupta, H., Zhou, Z., Das, S. R. and Gu, Q. (2003) 'Connected sensor cover: Self-organization of senor networks for efficient query execution', Proceedings of the 4th ACM international symposium on Mobile ad hoc networking and computing (MobiHoc), 2003, pp. 198-200.

Gupta, H., Zhou, Das, S. R. and Gu, Q. (2006) 'Connected sensor cover: Self-organization of senor networks for efficient query execution', IEEE/ACM Transactions on Networking (TON), Vol. 14, February, 2006, pp. 55-67.

Huang, C.-F. and Tseng, Y.-C. (2003) 'The coverage problem in a wireless sensor network', ACM International Conference Wireless Sensor Networks and Applications (WSNA), 2003.

Huang, C.-F. and Tseng, Y.-C. (2005) 'The coverage problem in a wireless sensor network', Mobile Networks and Applications, 2005. 
Hung, K.-S., Lui, K.-S. and Kwok, Y.-K. (2007) 'A Trust-based Geographical Routing Scheme in Sensor Networks', IEEE Wireless Communications and Networking Conference (WCNC), March, 2007.

Hung, K.-S. and Lui, K.-S. (2008) 'On Perimeter Coverage of Wireless Sensor Networks', Technical Report TR2008-004, Department of Electrical and Electronic Engineering, The University of Hong Kong, September, 2008.

Hung, K.-S. and Lui, K.-S. (2010a) 'On Perimeter Coverage of Wireless Sensor Networks', IEEE Transactions on Wireless Communications, To appear.

Hung, K.-S. (2010b) 'On Perimeter Coverage Issues in Wireless Sensor Networks', PhD Thesis, The University of Hong Kong, Feburary, 2010.

Ibarra, O. H., Wang, H. and Zheng Q. (1992) 'Minimum Cover and Single Source Shortest Path Problems for Weighted Interval Graphs and Circular-arc Graphs', Proceedings of Allerton, 1992.

Kar, K. and Banerjee, S. (2003) 'Node placement for connected coverage in sensor networks', Proceedings of WiOpt: Modeling and Optimization in Mobile, Ad Hoc and Wireless Networks, 2003, pp. 14-17.

Lee, C. C. and Lee, D. T. (1984) 'On a Circle-Cover Minimization Problem', Information Processing Letters, Elsevier Science Publishers, Vol. 18, February, 1984, pp. 109-115

Lee, H. and Aghajan, H. (2006) 'Vision-enabled node localization in wireless sensor networks.', COGnitive systems with Interactive Sensors (COGIS), March, 2006.

McCormick, C., Laligand, P.-Y., Lee, H. and Aghajan, H. (2006) 'Distributed agent control with self-localizing wireless image sensor networks.', COGnitive systems with Interactive Sensors (COGIS), March, 2006.

Olteanu, A., Xiao, Y., Wu. K. and Du, X. (2008) 'Weaving a Proper Net to Catch Large Objects.', IEEE Globecom, December, 2008. pp.133-137.

Osiris Photoelectric Sensors, http://www.schneiderelectric.ca/www/en/products/sensors2000/html/osiris.htm [Accessed 07/10/2009].

Shnayder, V., Hempstead, M., Chen, B., Allen, G. W. and Welsh, M. (2004) 'Simulating the Power Consumption of Large-Scale Sensor Network Applications', Proceedings of the 2nd international conference on Embedded networked sensor systems (ACM Sensys), November, 2004. pp. 188-200.

Shnayder, V., Hempstead, M., Chen, B., Allen, G. W. and Welsh, M. (2009) 'PowerTOSSIM: Efficient Power Simulation for TinyOS Applications', http://www.eecs.harvard.edu/_shnayder/ptossim, [Accessed 07/10/2009].

Tezcan N. and Wang, W. (2008) 'Self-Orienting Wireless Multimedia Sensor Networks for Maximizing Multimedia Coverage', IEEE International Conference on Communications (ICC 2008), May, 2008.

Thai, M. T., Li, Y., Wang, F. and Du, D.-Z. (2007) 'O(log $\mathrm{n})$-localized algorithms on the coverage problem in heterogeneous sensor networks', IEEE International Performance Computing and Communications Conference (IPCCC 2007), April, 2007. pp. 85-92.
Wang, J. and Medidi, S. (2007) 'Energy Efficient Coverage with Variable Sensing Radii in Wireless Sensor Networks', IEEE International Conference on Wireless and Mobile Computing, Networking and Communications (WiMob), 2007.

Wu, J. and Yang, S. (2004) 'Coverage and Connectivity in Sensor Networks with Adjustable Ranges', International Workshop on Mobile and Wireless Networking (MWN), August, 2004, pp. 188-200.

Ye, M., Chan, E., Chen, G. and Wu, J. (2006) 'Energy Efficient Fractional Coverage Schemes for Low Cost Wireless Sensor Networks', 26th IEEE International Conference on Distributed Computing Systems Workshops (ICDCS Workshops), 2006, pp. 7979 .

Yu, M. S., Chen, C. L. and Lee, R. C. T.(1989) 'Optimal parallel circle-cover and independent set algorithms for circular arc graphs', Proc. 1989 International Conference on Parallel Processing, 1989, pp. 126-129.

Zhou, Z., Das, S. and Gupta, H. (2004) 'Variable Radii Connected Sensor Cover in Sensor Networks', IEEE SECON, October, 2004. 